\title{
Metal-Insulator Transition in Two Dimensions
}

\author{
S. V. Kravchenko \\ Physics Department, Northeastern University, \\ Boston, Massachusetts 02115, U.S.A.
}

Received 27 May, 1999

\begin{abstract}
A metal-insulator transition was recently observed in a variety of two-dimensional electron and hole systems, contrary to the 20 year old belief that it is forbidden in two dimensions. The origin of this transition is still unknown and is being debated. In this paper, I briefly survey the main experimental findings and suggested explanations.
\end{abstract}

Until recently, it was believed that all twodimensional (2D) electron or hole systems are localized in the absence of a magnetic field in the limit of zero temperature no matter how clean the $2 \mathrm{D}$ system is. This conclusion was based on scaling theory for noninteracting electrons [1] and even in the case of weakly interacting particles [2]. The theory [1, 2] received experimental confirmation in a number of $2 \mathrm{D}$ materials (see, e.g., Ref.[3, 4]). However, measurements on very clean 2D systems suggested that a transition from insulating to conducting behavior occurs with increasing electron (hole) density, $n_{s}$, above some critical density, $n_{c}$ [5-12]. It was quickly realized that this transition occurs in the regime of high $r_{s}$ values where

$$
r_{s} \equiv \frac{E_{e e}}{E_{F}}=\frac{m^{*} e^{2}}{4\left(n_{s} \pi\right)^{1 / 2} \pi \hbar^{2} \epsilon \epsilon_{0}}
$$

is the ratio of the energy of electron-electron interactions and the Fermi energy (here $m^{*}$ is the effective mass and $\epsilon$ is the dielectric constant). In most 2D systems where this unexpected transition was found, $r_{s}$ values were of the order of 10 . Therefore, rather than being a small perturbation, correlations provide the main energy in the problem. Strongly interacting $2 \mathrm{D}$ systems are currently the focus of intense theoretical interest [13-20].

A typical temperature dependence of the resistivity of a high-quality 2D system (silicon metal-oxidesemiconductor field-effect transistor, "MOSFET") in a "dilute" (high $r_{s}$ ) regime is shown in Fig. 1 on a semilogarithmic scale. Different curves correspond to different electron densities which are set by the voltage applied between the gate and the $2 \mathrm{D}$ layer. The re- sistivity increases with decreasing temperature for the two lower electron densities indicating insulating ("normal") behavior. For the four highest $n_{s}$, the resistivity sharply decreases with decreasing temperature signaling a transition from insulating to conducting behavior. At a critical density $n_{c}=9.02 \times 10^{10} \mathrm{~cm}^{-1}$ the resistivity is practically temperature-independent. This temperature-independent point corresponds to the critical electron density for the metal-insulator transition.

Qualitatively the same $\rho(T)$ dependencies were recently obtained in other 2D systems: p-SiGe heterostructures [7], p-GaAs/AlGaAs heterostructures [8, 9], n-AlAs heterostructure [10], and n-GaAs/AlGaAs heterostructure [11]. A common feature of all these 2D systems is that the transition from insulating to apparent metallic behavior is seen at relatively high values of $r_{s}$. Another common feature is that the "critical" value of the resistivity at the transition, $\rho_{c} \equiv \rho\left(n_{c}\right)$, is of the order of the quantum of resistivity, $h / e^{2}$.

An important feature of the $\rho(T)$ dependencies around the metal-insulator transition is the fact that they can be "scaled" onto two distinct branches. In other words, the family of $\rho\left(n_{s}, T\right)$ curves can be represented as a function of $\delta / T^{b}$ where $\delta \equiv\left(n_{s}-n_{c}\right) / n_{c}$ and $b$ is a number of the order of 1 . An example of this scaling is shown in the inset to Fig. 1. The scaling analysis was first used in Ref.[5] and then successfully attempted in other systems $[6,7,9]$. However, in the cleanest systems, the data apparently fail to scale near the transition $[8,12]$. 


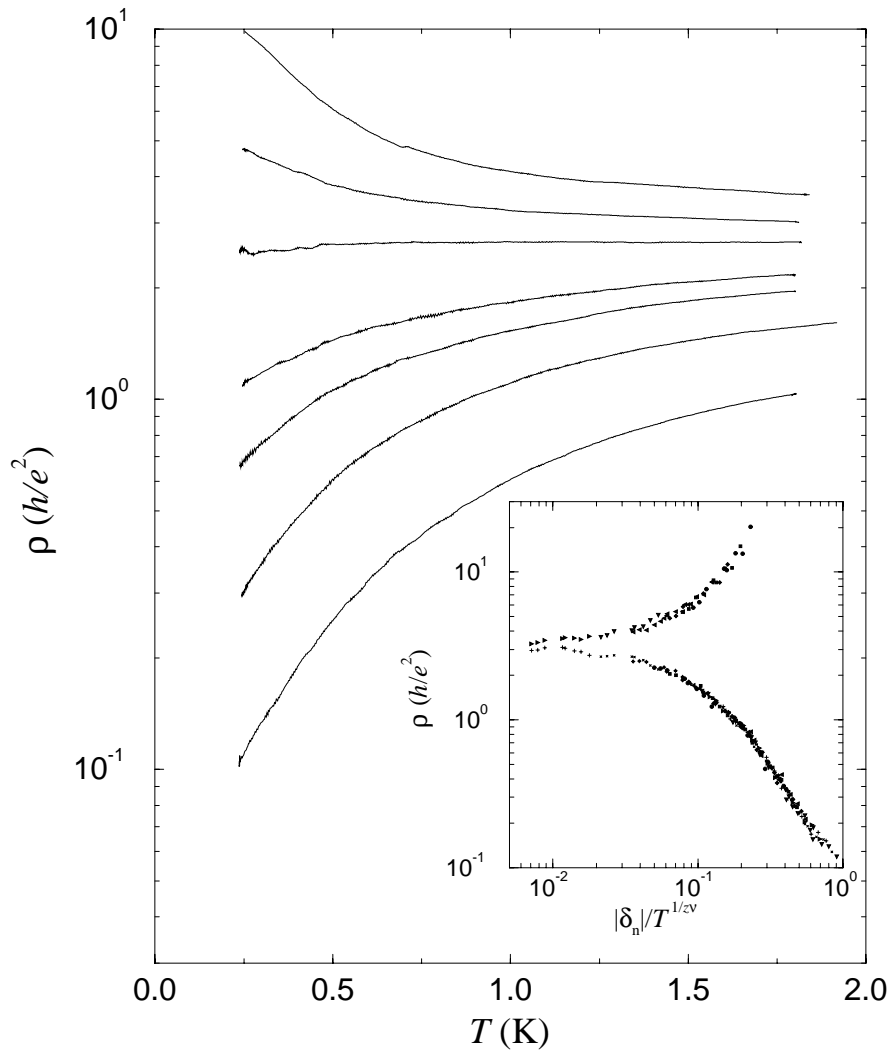

Figure 1. Resistivity of Si MOSFET in zero magnetic field as a function of temperature for electron densities $n_{s}=8.58,8.80,9.02,9.33,9.52,9.90$, and $1.10 \times 10^{10} \mathrm{~cm}^{-2}$. The critical density for the metal-insulator transition is $9.02 \times 10^{10} \mathrm{~cm}^{-2}$. The inset demonstrates scaling of the resistivity with temperature (after Ref. [5]).

One of the most interesting characteristics of the anomalous conducting phase is its dramatic response to a magnetic field applied parallel to the plane of the 2D system. An example of this behavior is shown in Fig. 2. The resistivity of silicon MOSFET increases by more than two orders of magnitude with increasing field, saturating to a new value in fields above $\sim 2$ Tesla [21, 22]. Apparently, the magnetic field drives a conducting system into an insulating state. However, in contrast with the $n_{s}$-driven metal-insulator transition, there appear to be no "critical" value of magnetic field below which the system remains conducting and above which it becomes insulating. In Ref.[21], a conclusion was made that a conducting state is suppressed by an arbitrary weak magnetic field. However, more recent measurements on a high-quality p-GaAs/AlGaAs suggest that such a critical magnetic field does exist [23]. This is currently an open question. We note that a parallel magnetic field couples only to the spins of the electrons and not to their orbital motion. Therefore, spins are believed to play a crucial role in the conducting phase.

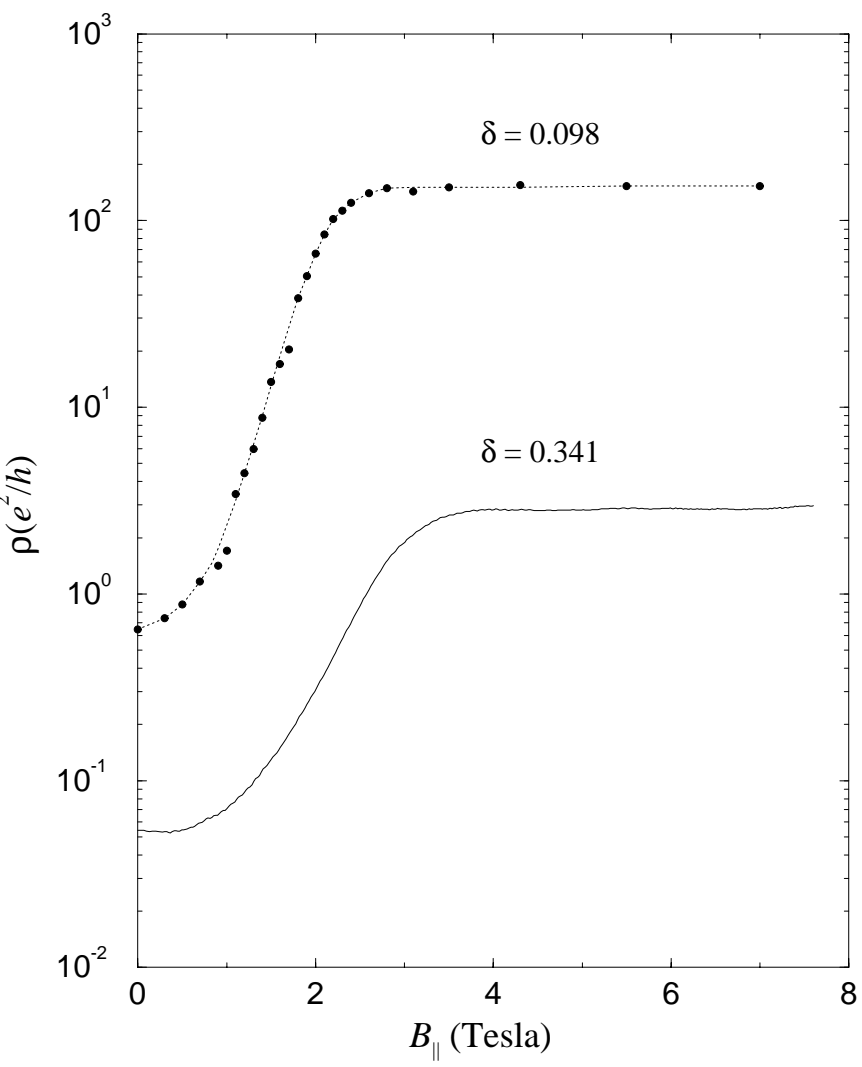

Figure 2. Resistivity of Si MOSFET as a function of parallel magnetic field on the metallic side of the transition at $T=0.25 \mathrm{~K}$. Upper curve: $\delta \equiv\left(n_{s}-n_{c}\right) / n_{c}=0.098$; lower curve: $\delta=0.341$.

Very recently, an interesting similarity in $\rho(T)$ between zero magnetic field case and an integer quantum Hall effect (QHE) state was observed in Si MOSFET [24]. As shown in Fig. 3, at both $B=0$ and at the Landau level filling factor $\nu=2, \rho(T)$ grows as the temperature is decreased, reaches a maximum around $T_{M} \approx 1 \mathrm{~K}$, and rapidly drops at lower temperatures. In contrast, the temperature dependence is very different at intermediate values of magnetic field, as shown by the dotted curve of Fig. 2 (a). In principle, the similarity between $\rho(T)$ at $B=0$ and $\nu=2$ may be accidental and simply due to the fact that the resistance in both cases decreases from a "classical" value above $T_{M}$ to a low-temperature "quantum" value. On the other hand, there may be a deeper connection between the $B=0$ and QHE states. A similarity between the metalinsulator transition in $B=0$ and the QHE-insulator transition was reported recently by Hanein et al.[25]. It was demonstrated that the QHE-insulator transition 
evolves smoothly and continuously to the $B=0$ transition discussed above. The critical resistivity at the QHE-insulator and $B=0$ metal-insulator transition were found to be surprisingly close to each other. These findings raise the possibility that the $B=0$ metalinsulator transition and QHE-insulator transition are physically related.

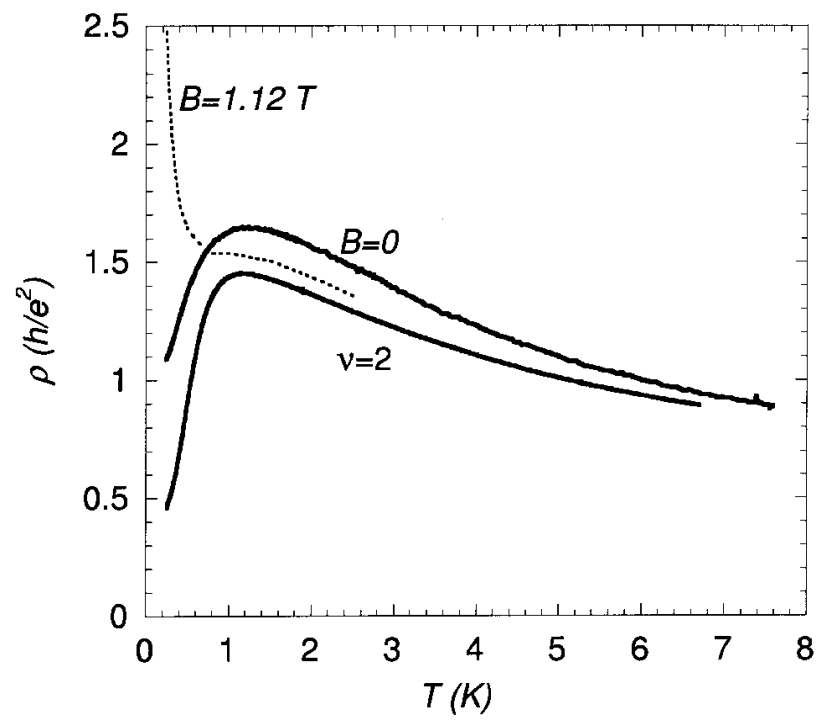

Figure 3. Temperature dependencies of the diagonal resistivity of Si MOSFET at $B=0$ and at Landau level filling factor $\nu=2$ for $n_{s}=0.74 \times 10^{11} \mathrm{~cm}^{-2}$. The dotted line shows the resistivity at $B=1.12$ Tesla.

Many theoretical explanations for the unanticipated $B=0$ metal-insulator transition were suggested recently [13-20]. Some of them $[13,14]$ are based on the pioneering work done by Finkelshtein in the early 1980 's where a metallic state was predicted in strongly interacting $2 \mathrm{D}$ systems. This state would be suppressed by a magnetic field in agreement with the experiment. However, the mechanism suggested in Ref.[13] predicts an onset for the metallic phase at temperatures that are lower than those found experimentally: in most systems, the drop in resistance is already seen at temperatures $\sim \frac{1}{3} E_{F} / k_{B}$, while in Ref. [13], the condition $T<</ k_{B} \tau<E_{F} / k_{B}$ was assumed (here $E_{F}$ is the Fermi energy and $\tau$ is the scattering time of the electron). This discrepancy was addressed in Ref.[14] where it was shown that the metal-insulator transition should be evident at temperatures of the order of the Fermi temperature. Other suggested models are based on non-Fermi liquid state $[16,17]$. Transition to an insulating behavior at $n_{s}<n_{c}$ is attributed in Ref.[17] to a formation of a Wigner solid. This prediction recently received a strong experimental support [12]. A possibility of having a "more traditional" non-Fermi liquid in 2D semiconductor-based systems - superconducting state - was also considered [18]. Recent findings of a critical magnetic field seem to support this possibility. Other possible explanations are based on strong spinorbit interaction [19]. There are also a few papers [20] in which the metal-insulator transition and other unusual properties of dilute 2D systems were attributed to classical effects such as temperature-dependent screening and charging/discharging of the ions which are known to exist close to the interface. The fact that despite all efforts, there is still no consensus in the theory suggests that there may be unusual new physics behind the $B=0$ metal-insulator transition in $2 \mathrm{D}$.

Experimental results shown in Figures 1-3 were obtained in collaboration with D. Simonian, M. P. Sarachik, K. Mertes, V. M. Pudalov, and T. M. Klapwijk. This work was supported by the National Science Foundation under Grant No. DMR9803440 .

\section{References}

[1] E. Abrahams, P. W. Anderson, D. C. Licciardello, and T. V. Ramakrishnan, Phys. Rev. Lett. 42, 673 (1979).

[2] B. L. Altshuler, A. G. Aronov, and P. A. Lee, Phys. Rev. Lett. 44, 1288 (1980).

[3] D. J. Bishop, D. C. Tsui, and R. C. Dynes, Phys. Rev. Lett. 44, 1153 (1980).

[4] M. J. Uren, R. A. Davies, M. Kaveh, and M. Pepper, J. Phys. C 44, 5737 (1981).

[5] S. V. Kravchenko et al., Phys. Rev. B 50, 8039 (1994); Phys. Rev. B 51, 7038 (1995); Phys. Rev. Lett. 77, 4938 (1996).

[6] D. Popovic, A. B. Fowler, and S. Washburn, Phys. Rev. Lett. 79, 1543 (1997).

[7] P. M. Coleridge, R. L. Williams, Y. Feng, and P. Zawadzki, Phys. Rev. B 56, R12764 (1997).

[8] Y. Hanein et al., Phys. Rev. Lett. 80, 1288 (1998).

[9] M. Y.Simmons et al., Phys. Rev. Lett. 80, 1292 (1998).

[10] S. J. Papadakis and M. Shayegan, Phys. Rev. B 57, R15068 (1998).

[11] Y. Hanein et al., Phys. Rev. B 58, R13338 (1998).

[12] J. Yoon, C. C. Li, D. Shahar, D. C. Tsui, and M. Shayegan, preprint cond-mat/9807235 (Phys. Rev. Lett., in press).

[13] A. M. Finkelshtein, Z. Phys. B 56, 189 (1984); C. Castellani, C. Di Castro, P. A. Lee, and M. Ma, Phys. Rev. B 30, 527 (1984); C. Castellani, C. D. Castro, and P. A. Lee, Phys. Rev. B 57, R9381 (1998)

[14] Q. Si and C. M. Varma, Phys. Rev. Lett. 81, 4951 (1998). 
[15] V. Dobrosavljevic, E. Abrahams, E. Miranda, and S. Chakravarty, Phys. Rev. Lett. 79, 455 (1997).

[16] S. Chakravarty, L. Yin, and E. Abrahams, Phys. Rev. B 58, R559 (1998).

[17] S. Chakravarty, S. Kivelson, C. Nayak, and K. Voelker, preprint cond-mat/9805383 (1998), submitted to $\mathrm{Na}$ ture (1998) (unpublished).

[18] P. Phillips et al., Nature 395, 253 (1998); D. Belitz and T. R. Kirkpatrick, Phys. Rev. B 58, 8214 (1998); J. S. Thakur and D. Neilson, Phys. Rev. B 58, 13717 (1998).

[19] Y. Lyanda-Geller, Phys. Rev. Lett. 80, 4273.

[20] S. He and X. C. Xie, Phys. Rev. Lett. 80, 3324 (1998); B. L. Altshuler and D. L. Maslov, Phys. Rev. Lett. 82, 145 (1999); T. M. Klapwijk and S. Das Sarma, preprint cond-mat/9810349 (1998) (Solid State Commun., in press); S. Das Sarma and E. H. Hwang, preprint condmat/9812216 (1998) (unpublished).

[21] D. Simonian, S. V. Kravchenko, M. P. Sarachik, and V. M. Pudalov, Phys. Rev. Lett. 79, 2304 (1997).

[22] V. M. Pudalov, G. Brunthaler, A. Prinz, and G. Bauer, JETP Lett. 65, 932 (1997).

[23] J. Yoon et al., abstract LC30.06 (1999 March Meeting of the APS).

[24] S. V. Kravchenko et al., preprint cond-mat/9812389 (1998) (submitted to Phys. Rev. B).

[25] Y. Hanein et al., preprint cond-mat/9901186 (1999) (submitted to Nature). 